Impact of limited solvent capacity on metabolic rate, enzyme activities and metabolite concentrations of $S$. cerevisiae glycolysis Protocol S1

Alexei Vazquez, Marcio Argollo de Menezes, Albert-László Barabási, and Zoltán N. Oltvai 
I. SCHEMATIC REPRESENTATION OF SACCHAROMYCES CEREVISIAE GLYCOLYSIS

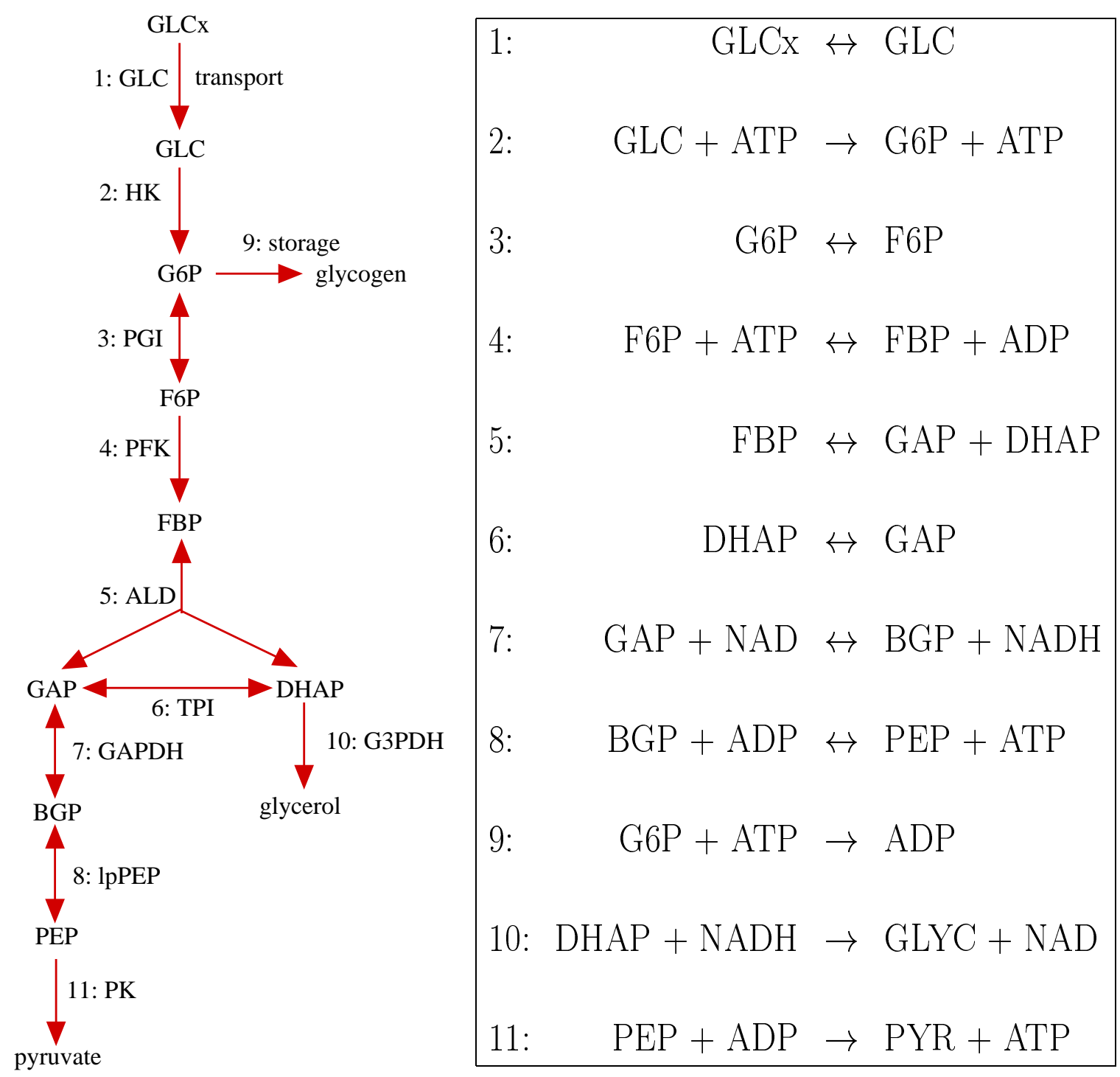




\section{MODEL AND MODEL PARAMETERS}

\section{A. Optimization objective}

The optimization objective is the glycolysis rate

$$
\frac{R}{1-\phi}=\frac{1}{\sum_{i=2}^{10} a_{i} r_{i}}
$$

where $\phi$ is the fraction of cell volume occupied by cell components other than glycolysis enzymes, $r_{i}$ is the rate of the $i$-th reaction relative to the glycolysis rate,

$$
a_{i}=\frac{v_{s p e c} \mu_{i} \rho}{x_{i} k_{i}}
$$

is the crowding coefficient associated with the $i$-th reaction, $v_{\text {spec }}$ is the specific volume, and $\mu_{i}$ and $k_{i}$ are the molar mass and catalytic constant of the enzyme catalyzing the $i$-th reaction. Note that the transport (3) and storage (11) reactions have been excluded. The former does not contribute to the cytoplasm crowding and the latter is considered and step outside glycolysis.

Given the storage rate and the concentration of ATP, the rate equation (13) below determines the concentration of G6P. Furthermore, given this G6P concentration, and the concentration of extracellular glucose, ATP and ADP, the rate equation (3) determines the concentration of intracellular glucose. The remaining metabolite concentrations are obtained such that to maximize (1).

\section{B. Rate equation models, as reported in Ref. [1]}

1: Glucose transport (TRANS)

$$
v_{1}=\frac{1+\frac{[G L C x]}{K_{1 G L C}}+\frac{P_{1} \frac{[G L C x]}{K_{1 G L C}}+1}{P_{1} \frac{G L C]}{K_{1 G L C}}+1}\left(1+\frac{[G L C]}{K_{1 G L C}}+\frac{[G 6 P]}{K_{1 I G L C}}+\frac{[G L C][G 6 P]}{K_{1 G L C} K_{1 I I G L C}}\right)}{\frac{[G L C x]-[G L C]}{K_{1 G L C}}} V_{1, \max }
$$

2: Hexokinase (HK)

$$
x_{2}=\frac{K_{2 D G L C} K_{2 A T P}+K_{2 G L C}[A T P]+K_{2 A T P}[G L C]+[G L C][A T P]}{[A T P][G L C]}
$$

3: Phosphoglucoisomerase (PGI)

$$
x_{3}=\frac{K_{3 G 6 P}+[G 6 P]+\frac{K_{3 G 6 P}}{K_{3 F 6 P}}[F 6 P]}{[G 6 P]-\frac{[F 6 P]}{K_{3 e q}}}
$$

4: Phosphofructokinase-1 (PFK)

$$
x_{4}=\frac{K_{5 F 6 P}\left(1+\kappa_{4} \frac{[A T P]^{2}}{[A D P]^{2}}\right)+\left([F 6 P]+\frac{K_{4 F 6 P}}{K_{4 F B P}}[F B P]\right)^{2}}{\left([F 6 P]-\frac{[F B P]}{K_{4 e q}}\right)\left([F 6 P]+\frac{K_{4 F 6 P}}{K_{4 F B P}}[F B P]\right)}
$$

This reaction is generally considered as irreversible. Ignoring its reversibility would result, however, in infinitely large values for FBP. Therefore, we have made the reversible extension of this model following [2].

5: Aldolase (ALD) 


$$
x_{5}=\frac{K_{5 F B P}+[F B P]+\frac{[G A P] K_{5 D H A P} V_{5 f}}{K_{5 e q} V_{5 r}}+\frac{[D H A P] K_{5 G A P} V_{5 f}}{K_{5 e q} V_{5 r}}+\frac{[F B P][G A P]}{K_{5 I G A P}}+\frac{[G A P][D H A P] V_{5 f}}{K_{5 e q} V_{5 r}}}{[F B P]-\frac{[G A P][D H A P]}{K_{5 e q}}}
$$

6: Triosephosphate isomerase (TPI)

$$
x_{6}=\frac{K_{6 D H A P}+[D H A P]+\frac{K_{6 D H A P}}{K_{6 G A P}}[G A P]}{[D H A P]-\frac{[G A P]}{K_{6 e q}}}
$$

7: Glyceraldehyde 3-phosphate dehydrogenase (GAPDH)

$$
x_{7}=\frac{K_{7 G A P} K_{7 N A D}\left(1+\frac{[G A P]}{K_{7 G A P}}+\frac{[B G P]}{K_{7 B G P}}\right)\left(1+\frac{[N A D]}{K_{7 N A D}}+\frac{[N A D H]}{K_{7 N A D H}}\right)}{[G A P][N A D]-\frac{[B G P][N A D H]}{K_{7 e q}}}
$$

8: (lpPEP)

$$
v_{8}=k_{8 f}[B G P][A D P]-k_{8 r}[P E P][A T P]
$$

9: Pyruvate kinase (PK)

$$
x_{9}=\frac{\left(K_{9 P E P}+[P E P]\right)\left(K_{9 A D P}+[A D P]\right)}{[A D P][P E P]}
$$

10: Glycerol 3-phosphate dehydrogenase (G3PDH)

$$
x_{10}=\frac{K_{10 D H A P}\left(1+\frac{K_{15 I N A D H}}{[N A D H]}\left(1+\frac{[N A D]}{K_{10 I N A D}}\right)\right)[D H A P]\left(1+\frac{K_{15 N A D H}}{[N A D H]}\left(1+\frac{[N A D]}{K_{10 N A D}}\right)\right)}{[D H A P]}
$$

11: Storage

$$
v_{11}=k_{11}[A T P][G 6 P]
$$


C. Kinetic constants, as reported in Ref. [1]

\begin{tabular}{|c|c|c|}
\hline Reaction & Parameter & Value \\
\hline 1: TRANS & $\begin{array}{l}K_{1 G L C} \\
K_{1 I G 6 P} \\
K_{1 I I G 6 P} \\
P_{1} \\
V_{1, \max } \\
\end{array}$ & $\begin{array}{l}1.7 \\
1.2 \\
7.2 \\
1 \\
1015 \mathrm{mM} / \mathrm{min} \\
\end{array}$ \\
\hline 2: HK & $\begin{array}{l}K_{2 A T P} \\
K_{3 G L C} \\
K_{3 D G L C} \\
\end{array}$ & \begin{tabular}{|l|}
0.1 \\
0 \\
0.37 \\
\end{tabular} \\
\hline 3: PGI & $\begin{array}{l}K_{3 G 6 P} \\
K_{3 F 6 P} \\
K_{3 e q} \\
\end{array}$ & \begin{tabular}{|l|}
0.8 \\
0.15 \\
0.13 \\
\end{tabular} \\
\hline 4: PFK & \begin{tabular}{|l|}
$K_{4 F 6 P}$ \\
$K_{4 F B P}$ \\
$\kappa_{4}$ \\
$K_{4 e q}$ \\
\end{tabular} & \begin{tabular}{|l|}
0.021 \\
$0.003^{a}$ \\
0.15 \\
$800.0^{b}$ \\
\end{tabular} \\
\hline 5: ALD & $\begin{array}{l}V_{5 f} / V_{5 r} \\
K_{5 F B P} \\
K_{5 G A P} \\
K_{5 D H A P} \\
K_{5 I G A P} \\
K_{5 e q} \\
\end{array}$ & $\begin{array}{l}0.2 \\
0.3 \\
4.0 \\
2.0 \\
10.0 \\
0.081 \\
\end{array}$ \\
\hline 6: TPI & $\begin{array}{l}K_{6 D H A P} \\
K_{6 G A P} \\
K_{6 e q} \\
\end{array}$ & $\begin{array}{l}1.23 \\
1.27 \\
0.055 \\
\end{array}$ \\
\hline 7: GAPDH & \begin{tabular}{|l}
$K_{7 G A P}$ \\
$K_{7 B G P}$ \\
$K_{7 N A D}$ \\
$K_{7 N A D H}$ \\
$K_{7 e q}$ \\
\end{tabular} & $\begin{array}{l}0.6 \\
0.01 \\
0.1 \\
0.06 \\
0.0055 \\
\end{array}$ \\
\hline 8: lpPEP & $\begin{array}{l}k_{8 f} \\
k_{8 r} \\
\end{array}$ & $\begin{array}{l}443900 \\
1529 \\
\end{array}$ \\
\hline 9: PK & \begin{tabular}{|l}
$K_{9 A D P}$ \\
$K_{9 P E P}$ \\
\end{tabular} & $\begin{array}{ll}0.17 \\
0.2 \\
\end{array}$ \\
\hline 10: G3PDH & \begin{tabular}{|l|}
$K_{10 N A D H}$ \\
$K_{10 D H A P}$ \\
$K_{10 I N A D H}$ \\
$K_{10 I N A D}$ \\
\end{tabular} & \begin{tabular}{|l|}
0.13 \\
25 \\
0.034 \\
0.13 \\
\end{tabular} \\
\hline 11: storage & $k_{11}$ & 2.26 \\
\hline
\end{tabular}

${ }^{a}$ This parameter was fitted to obtain the best agreement between the measured FBP concentration and the value predicted by the maximization of (1) with all other metabolite concentrations fixed to their experimentally determined values. ${ }^{b}$ From Ref. [4]. 


\section{Enzyme molar masses and catalytic constants}

\begin{tabular}{|l|l|l|l|}
\hline Nomenclature & Enzyme & Molar mass (g/mol) & Catalytic constant (1/s) \\
\hline$h k$ & hexokinase & 53738.7 & 96 \\
$p g i$ & phosphogluco isomerase & 61299.5 & 120 \\
$p f k$ & phospho-fructokinase & 107971 & 376 \\
ald & fructose 1,6-bisphosphate aldolase & 39620.9 & 142 \\
$t p i$ & triosephosphate isomerase & 26795.6 & 3580 \\
gapdh & D-glyceraldehyde 3-phosphate dehydrogenase & 35750 & 144 \\
$p k$ & pyruvate kinase & 55195.5 & 632 \\
g3pdh & glycerol 3-phosphate dehydrogenase & 42869.1 & 33.3 \\
\hline
\end{tabular}

The catalytic constants were obtained from experimental estimates for Saccharomyces carlsbergensis [3], except for g3pdh that was obtained from an estimate for Edidolon helvum [5].

\section{E. Specific volume and cell density}

\begin{tabular}{|l|l|l|l|}
\hline Parameter & Name & Value & Source \\
\hline$v_{\text {spec }}$ & Specific volume & $0.73 \mathrm{ml} / \mathrm{g}$ & globular proteins $[6]$ \\
$\rho$ & Cell density & $0.34 \mathrm{~g} / \mathrm{ml}$ & E. coli $[7]$ \\
\hline
\end{tabular}

F. Reaction rates, as reported in Ref. [1]

\begin{tabular}{|l|l|l|}
\hline Reaction/pathway & nomenclature & relative rate \\
\hline glycolysis & $v_{0}$ & $27 \mathrm{mM} / \mathrm{min}$ \\
fermentation & $r_{\text {ferm }}$ & 0.12 \\
glycerol production & $r_{\text {glyc }}$ & 0.13 \\
lactonitrile formation & $r_{\text {lact }}$ & 0.04 \\
glycogen buildup & $r_{\text {stor }}$ & 0.71 \\
HK & $r_{2}$ & $r_{\text {ferm }}+r_{\text {glyc }}+r_{\text {lact }}+2 r_{\text {stor }}$ \\
PGI & $r_{3}$ & $r_{\text {ferm }}+r_{\text {glyc }}+r_{\text {lact }}+r_{\text {stor }}$ \\
PFK & $r_{4}$ & $r_{\text {ferm }}+r_{\text {glyc }}+r_{\text {lact }}+r_{\text {stor }}$ \\
ALD & $r_{5}$ & $r_{\text {ferm }}+r_{\text {glyc }}+r_{\text {lact }}+r_{\text {stor }}$ \\
TPI & $r_{6}$ & $r_{\text {ferm }}+r_{\text {stor }}$ \\
GAPDH & $r_{7}$ & $2 r_{\text {ferm }}+r_{\text {glyc }}+r_{\text {lact }}+2 r_{\text {stor }}$ \\
lpPEP & $r_{8}$ & $2 r_{\text {ferm }}+r_{\text {glyc }}+r_{\text {lact }}+2 r_{\text {stor }}$ \\
PK & $r_{9}$ & $2 r_{\text {ferm }}+r_{\text {glyc }}+r_{\text {lact }}+2 r_{\text {stor }}$ \\
G3PDH & $r_{10}$ & $r_{\text {glyc }}+r_{\text {lact }}$ \\
\hline
\end{tabular}


G. Fixed metabolite concentrations, as reported in Ref. [1]

\begin{tabular}{|l|l|l|}
\hline Nomenclature & Metabolite & Experiment (mM) \\
\hline GLCx & External glucose & 1.6 \\
ATP & Adenosine 5'-triphosphate & 2.1 \\
ADP & Adenosine 5'-biphosphate & 1.5 \\
AMP & Adenosine 5'-monophosphate & 0.33 \\
NADH & Nicotinamide adenine dinucleotide & 0.33 \\
& (reduced form) & \\
NAD & Nicotinamide adenine dinucleotide & 0.65 \\
& (oxidized form) & \\
\hline
\end{tabular}

\section{EXPERIMENTAL DATA USED IN THE COMPARISON WITH THE THEORETICAL PREDICTIONS}

A. Metabolite concentrations, as reported in Ref. [1]

\begin{tabular}{|l|l|l|}
\hline Nomenclature & Metabolite & Concentration (mM) \\
\hline G6P & Glucose 6-phosphate & 4.1 \\
F6P & Fructose 6-phosphate & 0.5 \\
FBP & Fructose 1,6-biphosphate & 5.1 \\
GAP & Glyceraldehyde 3-phosphate & 0.12 \\
DHAP & Dihydroxyacetone phosphate & 2.5 \\
PEP & Phosphoenol pyruvate & 0.04 \\
\hline
\end{tabular}

B. Enzyme activities $A$ relative to the glycolysis rate $R$, as reported in [4]

\begin{tabular}{|l|l|l|}
\hline Nomenclature & Enzyme & Activity $(A / R)$ \\
\hline$p g i$ & phosphogluco isomerase & 3.15 \\
$p f k$ & phospho-fructokinase & 1.7 \\
ald & fructose 1,6-bisphosphate aldolase & 2.98 \\
tpi & triosephosphate isomerase & 21.0 \\
gapdh & D-glyceraldehyde 3-phosphate dehydrogenase & $11.0-60.0^{a}$ \\
$p k$ & pyruvate kinase & 10.1 \\
\hline
\end{tabular}

${ }^{a}$ For the forward and reverse reaction. In this case we used the average, 35.5, to make the comparison with the theoretical predictions. 


\section{SUBOPTIMAL METABOLITE CONCENTRATIONS}

\begin{tabular}{|l|l|l|l|}
\hline Metabolite & Experimen & Mean & SD \\
\hline g6p & 4.1 & 4.0 & fixed \\
f6p & 0.5 & 0.40 & 0.031 \\
fbp & 5.1 & 5.6 & 10 \\
gap & 0.12 & 0.12 & 0.013 \\
dhap & 2.5 & 2.5 & 0.11 \\
bgp & - & 0.00033 & 0.00012 \\
pep & 0.04 & 0.063 & 0.046 \\
\hline
\end{tabular}

This table shows the mean and standard deviation of metabolite concentrations for suboptimal glycolysis rates. We obtain these values from a Montecarlo sampling of the metabolite concentrations and weight the averages by the exponential factor $e^{\beta R}$. where $R$ is the glycolysis rate (which depends on metabolite concentrations) and $\beta$ is a control parameter. The latter weight is motivated by the fact that in an exponentially growing culture the cells abundance is proportional to $\exp$ (growth-ratextime) and the growth-rate is proportional to the glycolysis rate. We fix a value of $\beta$ resulting in an $R$ standard deviation of 10value we obtain the mean metabolite concentrations and standard deviations reported in the Supp. Table E. The mean of these suboptimal metabolite concentrations is in the range of the experimental values. And the standard deviations are of the order or smaller than the means. We add some text in the S. cerevisiae glycolysis section and the Supp. Table E addressing these observations. 
[1] Hynne F, Dano S, and Sorensen P G (2001) Full-scale model of glycolysis in Saccharomyces cerevisiae, Biophys Chem 94: 121-163

[2] Hofmeyr J-H and Cornish-Bowden A (1997) The reversible Hill equation: how to incorporate cooperative enzymes into metabolic models. Compt Appl Biosci 13: 377-385.

[3] Boiteux A and Hess B. (1981) Design of glycolysis, Phisl Trans R Soc Lond B 293: 5-22.

[4] Teusink B et al (2000) Can yeast glycolysis be understood in terms of in vitro kinetics of the constituent enzymes? testing biochemistry, Eur J Biochem 267: 5113-5329.

[5] Schomburg I, Chang A, Schomburg D (2002) BRENDA, enzyme data and metabolic information. Nucleic Acids Res 30: 47-49.

[6] Lee B (1983) Calculation of volume fluctuation for globular protein models. Proc Natl Acad Sci U S A 80: 622-626.

[7] Zimmerman SB, Trach SO (1991) Estimation of macromolecule concentrations and excluded volume effects for the cytoplasm of Escherichia coli. J Mol Biol 222: 599620. 